\title{
The Exact Distribution of the Condition Number of Complex Random Matrices
}

\author{
Lin Shi, ${ }^{1}$ Taibin Gan, ${ }^{2}$ Hong Zhu, ${ }^{1}$ and Xianming Gu${ }^{2}$ \\ ${ }^{1}$ School of Automation Engineering, University of Electronic Science and Technology of China, Chengdu, Sichuan 611731, China \\ ${ }^{2}$ School of Mathematical Sciences, University of Electronic Science and Technology of China, Chengdu, Sichuan 611731, China \\ Correspondence should be addressed to Lin Shi; shilinlavender@163.com
}

Received 19 August 2013; Accepted 24 September 2013

Academic Editors: E. A. Abdel-Salam and E. Francomano

Copyright (c) 2013 Lin Shi et al. This is an open access article distributed under the Creative Commons Attribution License, which permits unrestricted use, distribution, and reproduction in any medium, provided the original work is properly cited.

Let $G_{m \times n}(m \geq n)$ be a complex random matrix and $W=G_{m \times n}^{H} G_{m \times n}$ which is the complex Wishart matrix. Let $\lambda_{1}>\lambda_{2}>\ldots>\lambda_{n}>0$ and $\sigma_{1}>\sigma_{2}>\ldots>\sigma_{n}>0$ denote the eigenvalues of the $W$ and singular values of $G_{m \times n}$, respectively. The 2-norm condition number of $G_{m \times n}$ is $\kappa_{2}\left(G_{m \times n}\right)=\sqrt{\lambda_{1} / \lambda_{n}}=\sigma_{1} / \sigma_{n}$. In this paper, the exact distribution of the condition number of the complex Wishart matrices is derived. The distribution is expressed in terms of complex zonal polynomials.

\section{Introduction}

Over the past decade, multiple-input and multiple-output (MIMO) systems have been at the forefront of wireless communications research and development, due to their huge potential for delivering significant capacity compared with conventional systems [1-13]. The capacity and performance of practical MIMO transmission schemes are often dictated by the statistical eigenproperties of the instantaneous channel correlation matrix $W=G_{m \times n}^{H} G_{m \times n}$, where $G_{m \times n}$ is a complex Gaussian matrix and $W$ is known to follow a complex Wishart distribution.

In recent years, the statistical properties of Wishart matrices have been extensively studied and applied to a large number of MIMO applications. In statistics, the random eigenvalues are used in hypothesis testing, principal component analysis, canonical correlation analysis, multiple discriminant analysis, and so forth (see [7]). In nuclear physics, random eigenvalues are used to model nuclear energy levels and level spacing [6]. Moreover, the zeros of the Riemann zeta function are modeled using random eigenvalues [6]. Condition numbers arise in theory and applications of random matrices, such as multivariate statistics and quantum physics.

Let $G_{m \times n}(m \geq n)$ be a complex random matrix whose elements are independent and identically distributed (i.i.d.) standard normal random variables. As we know, the $n \times$ $n$ complex random matrix $W=G_{m \times n}^{H} G_{m \times n}$ is a complex Wishart matrix. Its distribution is denoted by $W \sim W(m, \Sigma)$, $\Sigma=\sigma^{2} I$. In addition, $W$ is a positive definite Hermitian matrix with real eigenvalues; let $\lambda_{1}>\lambda_{2}>\cdots>\lambda_{n}>0$ and $\sigma_{1}>\sigma_{2}>\cdots>\sigma_{n}>0$ denote the eigenvalues of the $W$ and singular values of $G_{m \times n}$, respectively. The 2-norm condition number of $G_{m \times n}$ is $\kappa_{2}\left(G_{m \times n}\right)=\sqrt{\lambda_{1} / \lambda_{n}}=\sigma_{1} / \sigma_{n}$; thus, $\kappa_{2}(W)=\kappa_{2}\left(G_{m \times n}\right)^{2}$.

The exact distributions of the condition number of a $2 \times n$ matrix whose elements are independent and identically distributed standard normal real or complex random variables are given in [5] by Edelman. Edelman also obtained the limiting distributions and the limiting expected logarithms of the condition numbers of random rectangular matrices whose elements are independent and identically distributed standard normal random variables. The exact distributions of the condition number of Gaussian matrices are studied in [1] for real random matrix. Here, we derive the exact distribution of the condition number of a complex random matrix for special case $\Sigma=\sigma^{2} I$.

This paper is arranged as follows. Section 2 gives some preliminary results to the complex random matrices. In Section 3, the main result of this work, the density function of $\kappa_{2}\left(W_{n \times n}\right)$ for complex case, is proved. 


\section{Some Preliminary Results}

In this section, we give some results on joint density of the eigenvalues of a complex Wishart matrix $W$ and $W \sim W(m, \Sigma)$, $\Sigma=\sigma^{2} I$. The determinant, trace, and norm of a square matrix $B$ are denoted by $|B|, \operatorname{tr}(B)$, and $\|B\|$, respectively.

For any nonnegative integer $p$, a portion of $p$ is a multiple $\left(a_{1}, a_{2}, \ldots, a_{n}\right)$, where $a_{1} \geq a_{2} \geq \cdots \geq a_{n} \geq 0$ such that $\sum_{i=1}^{n} a_{i}=p, \mathscr{D}_{p}$ is the set of all portions of $p$, and the symbol $\sum_{p} a_{\kappa}$ means the summation over $\mathscr{D}_{p}$; that is, $\sum_{p} a_{\kappa}=$ $\sum_{\kappa \in \mathscr{D}_{p}} a_{\kappa}$.

Let $\kappa$ be any portion of $p$, and let $\mu_{1}>\mu_{2}>\cdots>\mu_{n}$ be the eigenvalues of an $n \times n$ matrix $B$ as follows:

$$
\begin{gathered}
\alpha_{[\kappa]}=p ! \frac{\prod_{i<j}^{n}\left(a_{i}-a_{j}-i+j\right)}{\prod_{i<j}^{n}\left(a_{i}+n-i\right) !}, \\
\alpha_{\kappa}(B)=\frac{\left|\left[\left(\mu_{i}^{a_{j}+n-j}\right)\right]\right|}{\left|\left[\left(\mu_{i}^{n-j}\right)\right]\right|} .
\end{gathered}
$$

The zonal polynomials (also called Schur polynomials) of $B$ are defined by

$$
C_{\kappa}(B)=\alpha_{[\kappa]} \cdot \alpha_{[\kappa]}(B)
$$

The following is the basic properties of the zonal polynomials [8]:

$$
[\operatorname{tr}(B)]^{p}=\sum_{p} C_{\kappa}(B)=\sum_{\kappa \in \mathscr{D}_{p}} C_{\kappa}(B)
$$

The zonal polynomial of the identity matrix is defined by

$$
C_{\kappa}\left(I_{n}\right)=2^{2 p} p !\left[\frac{1}{2} n\right]_{\kappa} \frac{\prod_{i<j}^{r}\left(2 a_{i}-2 a_{j}-i+j\right)}{\prod_{1}^{r}\left(2 a_{i}+r-i\right) !},
$$

where

$$
\begin{gathered}
{\left[\frac{1}{2} n\right]_{\kappa}=\prod_{i=1}^{n}\left(\frac{1}{2}(n-i+1)\right)_{a_{i}}} \\
(x)_{a_{i}}=x(x+1) \cdots\left(x+a_{i}-1\right), \quad(x)_{0}=1 .
\end{gathered}
$$

Definition 1. For $W=G_{m \times n}^{H} G_{m \times n}$, where $W$ is positive definite Hermitian matrix with real eigenvalues, let $G_{m \times n}$ be a complex random matrix whose elements are independent and identically distributed (i.i.d.) standard normal random variables. Let $\lambda_{1}>\lambda_{2}>\cdots>\lambda_{n}>0$ be the eigenvalues of $W$ and $\Lambda=\operatorname{diag}\left(\lambda_{1}, \lambda_{2}, \ldots, \lambda_{n}\right)$. If $W \sim W(m, \Sigma), \Sigma=\sigma^{2} I$ with $m \geq n$, then the joint density of its eigenvalues is defined [2] as follows:

$$
\begin{aligned}
f(\Lambda)= & \frac{\pi^{n(n-1)}\left(\sigma^{2}\right)^{-m n}}{\Gamma_{n}(n) \Gamma_{n}(m)} \\
& \times \prod_{i=1}^{n} \lambda_{i}^{m-n} \prod_{i<l}^{n}\left(\lambda_{i}-\lambda_{l}\right)^{2} \exp \left(-\frac{1}{\sigma^{2}} \sum_{i=1}^{n} \lambda_{i}\right),
\end{aligned}
$$

where

$$
\begin{gathered}
\Gamma_{n}(a)=\pi^{n(n-1) / 2} \prod_{i=1}^{n} \Gamma(a+k+1), \\
\Gamma(x)=\int_{0}^{+\infty} t^{x-1} e^{-t} d t .
\end{gathered}
$$

Lemma 2. For an $n \times n$ matrix $B$, the product of two zonal polynomials can be expressed in terms of a weighted combination of another zonal polynomial [7]; that is, for all $\nu \in \mathscr{D}_{s}$ and $\forall \kappa \in \mathscr{D}_{p}$, one has

$$
C_{v}(B) C_{\kappa}(B)=\sum_{\tau \in \mathscr{D}_{t}} g_{v, \kappa}^{\tau} C_{\tau}(B)
$$

where $t=s+p$ and $g_{v, \kappa}^{\tau}$ is a constant coefficient.

Lemma 3. For an $m \times n$ matrix $B$ and for all $\kappa \in \mathscr{D}_{p}$, one has [1]

$$
|I-B|^{m-n} C_{\kappa}(B)=\frac{\sum_{s=0}^{\infty} \sum_{\nu \in \mathscr{D}_{s}} \sum_{\tau \in \mathscr{D}_{t}}(n-m)_{\nu} g_{\nu, \kappa}^{\tau} C_{\tau}(B)}{s !}
$$

where $t=s+p$ and $g_{v, \kappa}^{\tau}$ is a constant coefficient.

Lemma 4. Let $Z=\operatorname{diag}\left(\xi_{2}, \ldots, \xi_{n}\right), Z_{1}=\operatorname{diag}\left(1, \xi_{2}, \ldots, \xi_{n}\right)$ and $D=\left\{1>\xi_{2}>\cdots>\xi_{n}>0\right\}$, then [2]

$$
\begin{gathered}
\int_{D}|Z|^{b-n} \prod_{i=2}^{n}\left(1-\xi_{i}\right)^{2} \prod_{i<l}^{n}\left(\xi_{i}-\xi_{l}\right)^{2} C_{\kappa}\left(Z_{1}\right) \prod_{i=2}^{n} d \xi_{i} \\
=(n b+p) \frac{\Gamma_{n}(n)}{\pi^{n(n-1)}} \frac{\Gamma_{n}(b, \kappa) \Gamma_{n}(n)}{\Gamma_{n}(b+n, \kappa)} C_{\kappa}(I),
\end{gathered}
$$

where

$$
\begin{gathered}
\Gamma_{n}(a)=\pi^{n(n-1) / 2} \prod_{i=1}^{n} \Gamma(a+k+1), \\
\Gamma_{n}(b, \kappa)=\pi^{n(n-1) / 2} \prod_{i=1}^{n} \Gamma\left(a+k_{i}-i+1\right) .
\end{gathered}
$$

\section{Main Result}

The exact distribution of the 2-norm condition number of the Wishart matrix $W$ is derived by the following.

Theorem 5. Let $G_{m \times n}$ be a complex random matrix and $W=$ $G_{m \times n}^{H} G_{m \times n} . \lambda_{1}$ and $\lambda_{n}$ are the maximum and minimum 
eigenvalues of $W, \kappa_{2}(W)=\lambda_{1} / \lambda_{n}$. Then, the exact distribution of $\kappa_{2}(W)$ is given by

$$
\begin{aligned}
f\left(\kappa_{2}(W)\right)= & \frac{\pi^{n(n-1)}\left(\sigma^{2}\right)^{-m n}}{\Gamma_{n}(n) \Gamma_{n}(m)} \exp \left(-\frac{n}{\sigma^{2}} \lambda_{1}\right) \\
& \times \sum_{p=0}^{\infty} \sum_{\kappa \in \mathscr{D}_{p}} \frac{\lambda_{1}^{m n-n+p}}{\sigma^{2 p} p !} \sum_{s=0}^{\infty} \sum_{\nu \in \mathscr{D}_{s}} \sum_{\tau \in \mathscr{D}_{t}} \frac{1}{s !}(n-m)_{\nu} \\
& \times g_{\gamma, \kappa}^{\tau} C_{\tau}\left(\kappa_{2}(W)-1\right)^{(n-1)(n+1)+p+s-1} \\
& \times \kappa_{2}(W)^{-(n-1)(n+1)-p-s+1} \times\left(n^{2}-1+p+s\right) \\
& \times \frac{\Gamma_{n-1}(n-1)}{\pi^{(n-1)(n-2)}} \\
& \times \frac{\Gamma_{n-1}(n+1, \tau) \Gamma_{n-1}(n-1)}{\Gamma_{n-1}(2 n, \tau)} C_{\tau}(I),
\end{aligned}
$$

where $t=s+p$.

Proof (consider (6)). By making the transformation $\lambda_{1}=\lambda_{1}$, $\gamma_{i}=1-\lambda_{i} / \lambda_{1}, i=2, \ldots, n$, where $1>\gamma_{n}>\cdots>\gamma_{2}>0$, we obtain the joint distribution of $\lambda_{1}$ and $\gamma_{2}, \ldots, \gamma_{n}$, as follows:

$$
\begin{aligned}
& \frac{\pi^{n(n-1)}\left(\sigma^{2}\right)^{-m n}}{\Gamma_{n}(n) \Gamma_{n}(m)} \exp \left(-\frac{n}{\sigma^{2}} \lambda_{1}\right) \lambda_{1}^{m n-n}|A|^{2}|I-A|^{m-n} \\
& \quad \times \prod_{k<l}^{n}\left(\gamma_{k}-\gamma_{k}\right)^{2} \exp \left[\frac{\lambda_{1}}{\sigma^{2}} \operatorname{tr}(A)\right] .
\end{aligned}
$$

By using Taylor’s formula,

$$
\begin{aligned}
& \frac{\pi^{n(n-1)}\left(\sigma^{2}\right)^{-m n}}{\Gamma_{n}(n) \Gamma_{n}(m)} \exp \left(-\frac{n}{\sigma^{2}} \lambda_{1}\right) \lambda_{1}^{m n-n}|A|^{2}|I-A|^{m-n} \\
& \quad \times \prod_{k<l}^{n}\left(\gamma_{k}-\gamma_{k}\right)^{2} \sum_{p=0}^{\infty} \frac{1}{p !}\left[\frac{\lambda_{1}}{\sigma^{2}} \operatorname{tr}(A)\right]^{p} .
\end{aligned}
$$

By using property (3), we have

$$
\begin{aligned}
& \frac{\pi^{n(n-1)}\left(\sigma^{2}\right)^{-m n}}{\Gamma_{n}(n) \Gamma_{n}(m)} \exp \left(-\frac{n}{\sigma^{2}} \lambda_{1}\right)|A|^{2}|I-A|^{m-n} \\
& \quad \times \prod_{i<l}^{n}\left(\gamma_{i}-\gamma_{l}\right)^{2} \sum_{p=0}^{\infty} \sum_{\kappa \in \mathscr{D}_{p}} \frac{\lambda_{1}^{m n-n+p} C_{\kappa}(A)}{\sigma^{2 p} p !},
\end{aligned}
$$

where $A=\operatorname{diag}\left(\gamma_{2}, \ldots, \gamma_{n}\right)$.
By making the transformation $\xi_{i}=\gamma_{i} / \gamma_{n}, i=2, \ldots, n-1$, using Lemma 3 , and integrating over the set $1>\xi_{n-1}>\cdots>$ $\xi_{2}>0$, we have

$$
\begin{gathered}
\int_{1>\gamma_{n-1}>\cdots>\gamma_{2}>0}|A|^{2}|I-A|^{m-n} C_{\kappa}(A) \prod_{i<l}^{n}\left(\gamma_{i}-\gamma_{l}\right)^{2} \prod_{i=2}^{n-1} d \gamma_{i} \\
=\left[\sum_{s=0}^{\infty} \sum_{\nu \in \mathscr{S}_{s}} \sum_{\tau \in \mathscr{D}_{t}} \frac{1}{s !}(n-m)_{\nu} g_{\nu, \kappa}^{\tau} \gamma_{n}^{(n-1)(n-2)+p+s-1}\right] \\
\times \int_{1>\xi_{n-1}>\cdots>\xi_{2}>0}|Z|^{2} C_{\tau}\left(Z_{1}\right) \\
\times \prod_{i=2}^{n-1}\left(1-\xi_{i}\right)^{2} \prod_{i, j=2}^{n-1}\left(\xi_{i}-\xi_{j}\right)^{2} \prod_{i=2}^{n-1} d \xi_{i},
\end{gathered}
$$

where $Z=\operatorname{diag}\left(\xi_{2}, \ldots, \xi_{n-1}\right)$ and $Z_{1}=\operatorname{diag}(1, Z)$.

By using Lemma 4 , let $n$ be replaced by $n-1$, let $\kappa$ be replaced by $\tau$, and let $b=n+1$.

Then,

$$
\begin{gathered}
\int_{1>\xi_{n-1}>\cdots, \xi_{2}>0}|Z|^{2} C_{\tau}\left(Z_{1}\right) \\
\times \prod_{i=2}^{n-1}\left(1-\xi_{i}\right)^{2} \prod_{i, j=2}^{n-1}\left(\xi_{i}-\xi_{j}\right)^{2} \prod_{i=2}^{n-1} d \xi_{i} \\
=\left(n^{2}-1+p+s\right) \frac{\Gamma_{n-1}(n-1)}{\pi^{n(n-1)}} \\
\times \frac{\Gamma_{n-1}(n+1, \tau) \Gamma_{n-1}(n-1)}{\Gamma_{n-1}(2 n, \tau)} C_{\tau}(I) .
\end{gathered}
$$

It follows that the distribution of $\left(\lambda_{1}, \gamma_{n}\right)$ is given by

$$
\begin{aligned}
f\left(\lambda_{1}, \gamma_{n}\right)= & \frac{\pi^{n(n-1)}\left(\sigma^{2}\right)^{-m n}}{\Gamma_{n}(n) \Gamma_{n}(m)} \exp \left(-\frac{n}{\sigma^{2}} \lambda_{1}\right) \\
& \times \sum_{p=0}^{\infty} \sum_{\kappa \in \mathscr{D}_{p}} \frac{\lambda_{1}^{m n-n+p}}{\sigma^{2 p} p !} \sum_{s=0}^{\infty} \sum_{v \in \mathscr{D}_{s}} \sum_{\tau \in \mathscr{D}_{t}} \frac{1}{s !}(n-m)_{v} \\
& \times g_{\gamma, \kappa}^{\tau} \gamma_{n}^{(n-1)(n+1)+p+s-1}\left(n^{2}-1+p+s\right) \\
& \times \frac{\Gamma_{n-1}(n-1)}{\pi^{(n-1)(n-2)}} \frac{\Gamma_{n-1}(n+1, \tau) \Gamma_{n-1}(n-1)}{\Gamma_{n-1}(2 n, \tau)} C_{\tau}(I) .
\end{aligned}
$$


Note that $\kappa_{2}(W)=\lambda_{1} / \lambda_{n}=\left(1-\gamma_{n}\right)^{-1}$; then, the distribution of $\kappa_{2}(W)=\left(1-\gamma_{n}\right)^{-1}$ is given by

$$
\begin{aligned}
f\left(\kappa_{2}(W)\right)= & \frac{\pi^{n(n-1)}\left(\sigma^{2}\right)^{-m n}}{\Gamma_{n}(n) \Gamma_{n}(m)} \exp \left(-\frac{n}{\sigma^{2}} \lambda_{1}\right) \\
& \times \sum_{p=0}^{\infty} \sum_{\kappa \in \mathscr{D}_{p}} \frac{\lambda_{1}^{m n-n+p}}{\sigma^{2 p} p !} \sum_{s=0}^{\infty} \sum_{\nu \in \mathscr{D}_{s}} \sum_{\tau \in \mathscr{D}_{t}} \frac{1}{s !}(n-m)_{v} \\
& \times g_{\nu, \kappa}^{\tau} C_{\tau}\left(\kappa_{2}(W)-1\right)^{(n-1)(n+1)+p+s-1} \\
& \times \kappa_{2}(W)^{-(n-1)(n+1)-p-s+1} \times\left(n^{2}-1+p+s\right) \\
& \times \frac{\Gamma_{n-1}(n-1)}{\pi^{(n-1)(n-2)}} \\
& \times \frac{\Gamma_{n-1}(n+1, \tau) \Gamma_{n-1}(n-1)}{\Gamma_{n-1}(2 n, \tau)} C_{\tau}(I) .
\end{aligned}
$$

\section{Conclusions}

In this paper, the exact distribution of the condition number of complex Wishart matrices is derived. The distribution is expressed in terms of complex zonal polynomials. This distribution plays an important role in numerical analysis and statistical hypothesis testing.

\section{Acknowledgments}

The authors would like to thank the anonymous reviewers for their detailed comments and suggestions. This work was supported by the Fund of Sichuan Provincial Key Laboratory of Signal and Information Processing, Xihua University (SZJJ2009-002).

\section{References}

[1] W. Anderson and M. T. Wells, "The exact distribution of the condition number of a gaussian matrix," SIAM Journal on Matrix Analysis and Applications, vol. 31, no. 3, pp. 1125-1130, 2009.

[2] T. Ratnarajah, R. Vaillancourt, and M. Alvo, "Eigenvalues and condition numbers of complex random matrices," SIAM Journal on Matrix Analysis and Applications, vol. 26, no. 2, pp. 441-456, 2005.

[3] A. T. James, "The distribution of the latent roots of the covariance matrix," Annals of Mathematical Statistics, vol. 31, pp. 547-560, 1960.

[4] Z. Chen and J. J. Dongarra, "Condition numbers of Gaussian random matrices," SIAM Journal on Matrix Analysis and Applications, vol. 27, no. 3, pp. 603-620, 2005.

[5] A. Edelman, "Eigenvalues and condition numbers of random matrices," SIAM Journal on Matrix Analysis and Applications, vol. 9, no. 4, pp. 543-560, 1988.
[6] M. L. Mehta, Random Matrices, Academic Press, New York, NY, USA, 2nd edition, 1991.

[7] R. J. Muirhead, Aspects of Multivatiate Statistical Theory, 1982.

[8] A. T. James, "Distributions of matrix variate and latent roots derived from normal samples," Annals of Mathematical Statistics, vol. 27, pp. 475-501, 1964.

[9] M. Matthaiou, M. R. McKay, P. J. Smith, and J. A. Nossek, "On the condition number distribution of complex wishart matrices," IEEE Transactions on Communications, vol. 58, no. 6, pp. 1705-1717, 2010.

[10] M. Kang and M. S. Alouini, "Largest eigenvalue of complex wishart matrices and performance analysis of MIMO MRC systems," IEEE Journal on Selected Areas in Communications, vol. 21, no. 3, pp. 418-426, 2003.

[11] S. Jin, M. R. Mckay, X. Gao, and I. B. Collings, "MIMO multichannel beamforming: SER and outage using new eigenvalue distributions of complex noncentral Wishart matrices," IEEE Transactions on Communications, vol. 56, no. 3, pp. 424-434, 2008.

[12] A. Zanella, M. Chiani, and M. Z. Win, "On the marginal distribution of the eigenvalues of wishart matrices," IEEE Transactions on Communications, vol. 57, no. 4, pp. 1050-1060, 2009.

[13] M. Matthaiou, D. I. Laurenson, and C. X. Wang, "On analytical derivations of the condition number distributions of dual non-central Wishart matrices," IEEE Transactions on Wireless Communications, vol. 8, no. 3, pp. 1212-1217, 2009. 


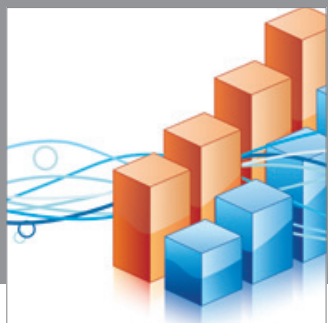

Advances in

Operations Research

mansans

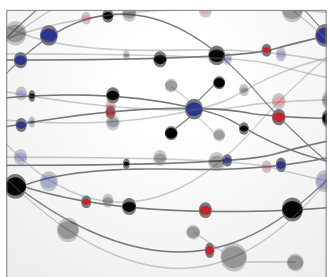

The Scientific World Journal
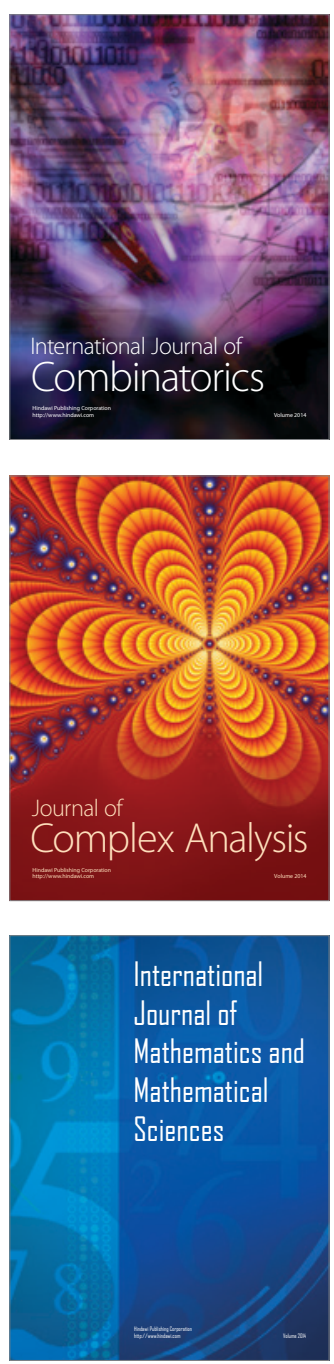
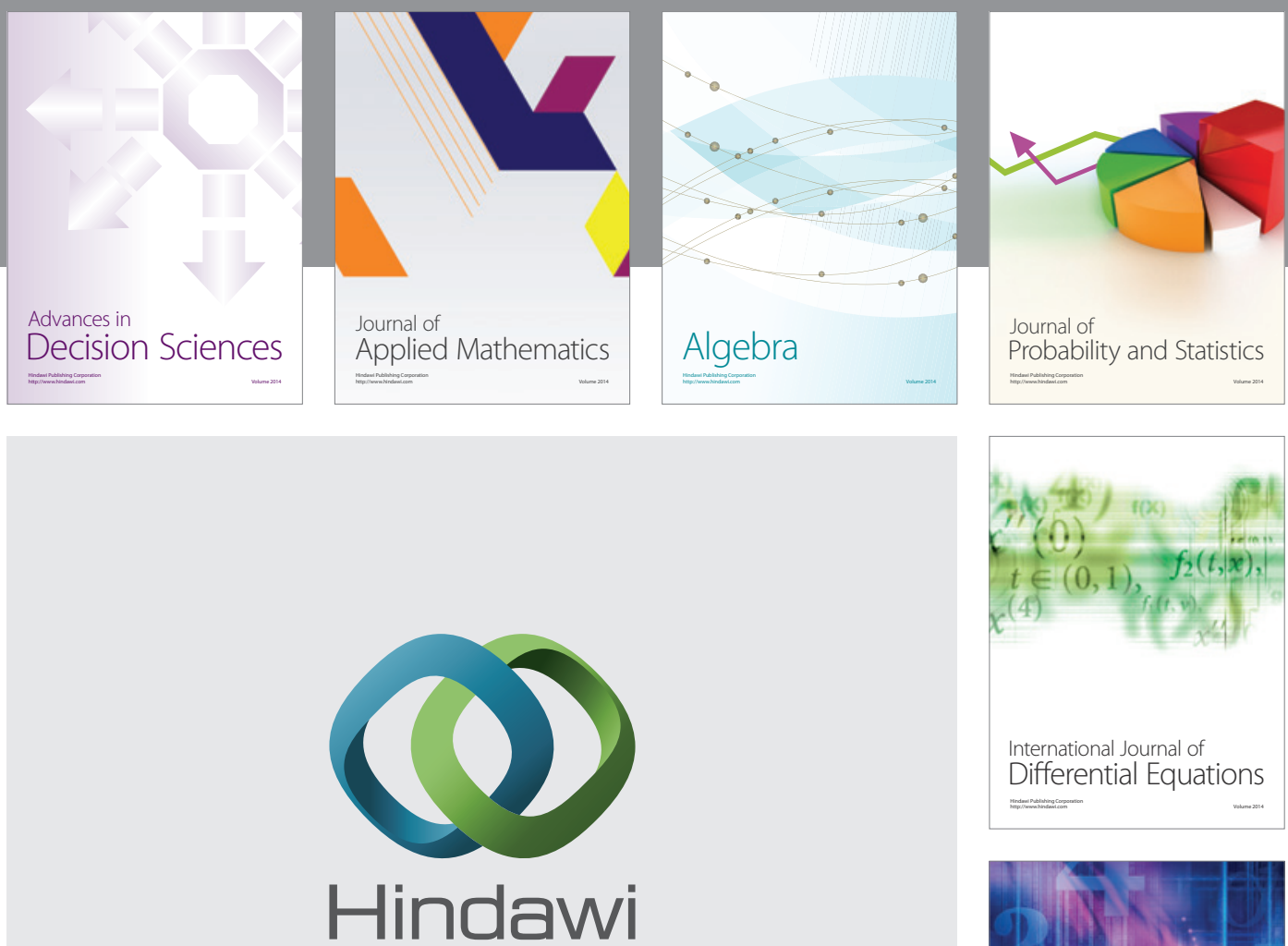

Submit your manuscripts at http://www.hindawi.com
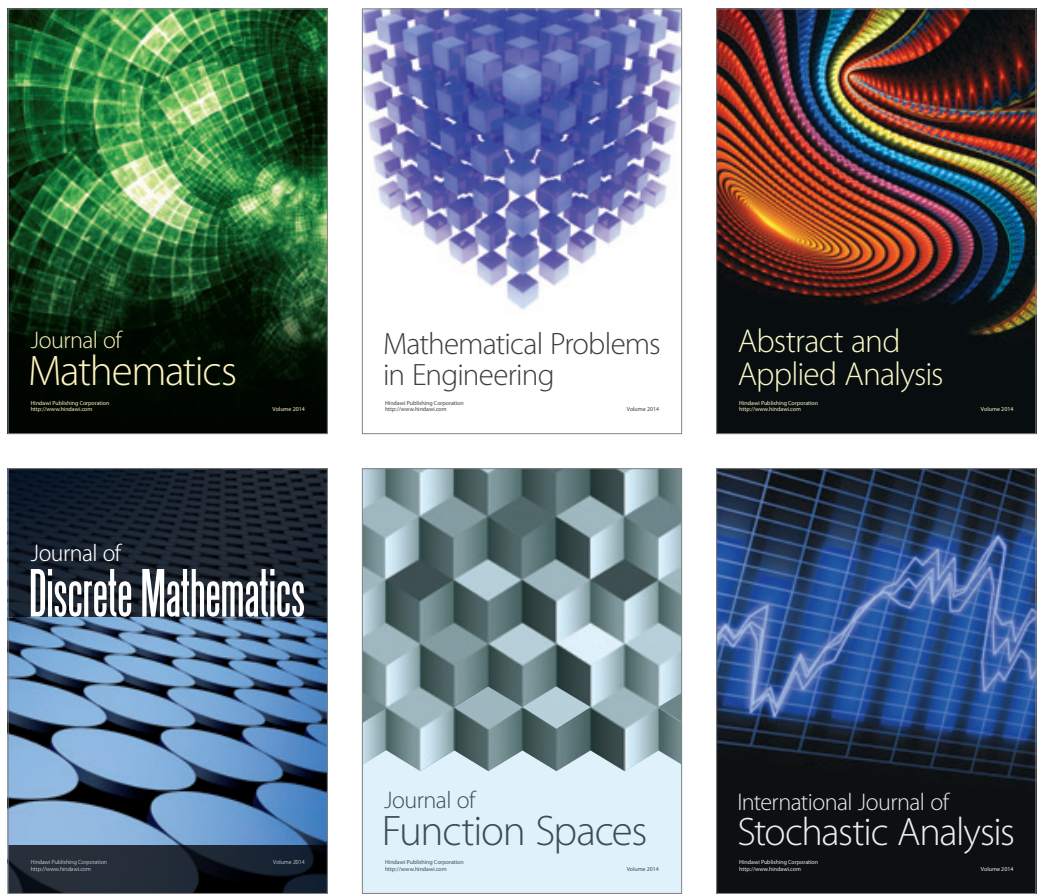

Journal of

Function Spaces

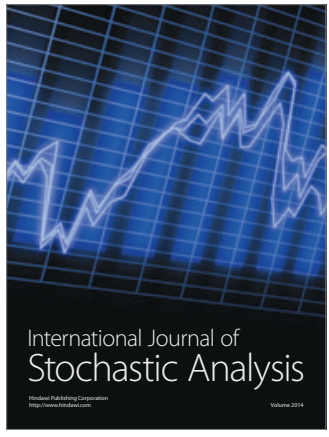

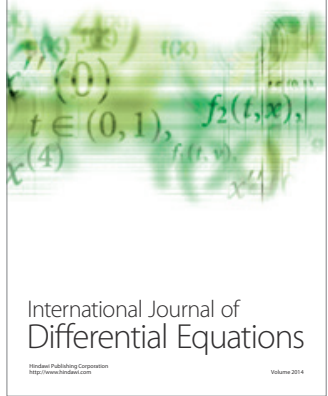
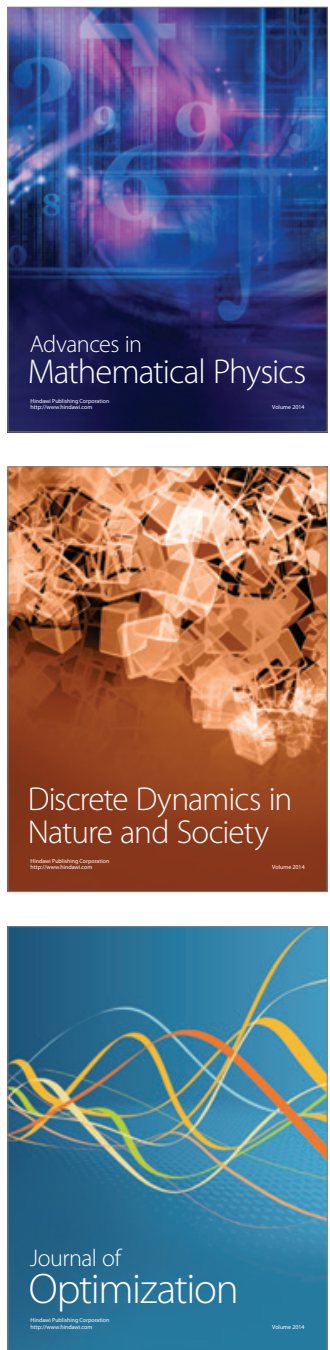